\title{
Natural Area Coding Based Postcode Scheme
}

\author{
Valentin Rwerekane ${ }^{1 *}$, Maurice Ndashimye ${ }^{2}$ \\ 1 Department of Computer Science, University of Rwanda, Huye, Rwanda. \\ 2 iThemba Labs, University of South Africa, Cape Town, South Africa. \\ * Corresponding author. Tel.: +250 788873 955; email: maurice@aims.ac.za \\ Manuscript submitted March 7th, 2017; accepted June 23, 2017. \\ doi: 10.177606/ijcce.6.3.161-172
}

\begin{abstract}
Traditionally, addresses were used to direct people and helped in social activities; nowadays addresses are used in a wide range of applications, such as automated mail processing, vehicles navigation, urban planning and maintenance, emergency response, statistical analyses, marketing, and others, to ensure necessities induced by new information technologies and facility developments. On top of addresses primary use, postcodes systems were developed to comprehensively provide a variety of public and commercial services. Postcode being an integral part of an addressing system, if well-established, a postcode system brings further social-economic development benefits to a country.

This paper aims at designing a postcode based on the Natural Area Coding (NAC). The design focuses on designing a standardized postcode that can fit into any addressing scheme and be used for towns and cities of any shapes from structured cities to slums.

Design considerations of a fine-grained postcode (easy for humans, efficient for computerized systems and requiring less or no maintenance over time to improve its efficiency) have proven to be difficult to realize. Therefore, in this paper a new logic is illustrated whereby these considerations are rationally handled while simultaneously allowing the postcode to give a sense of directions and distance. The technique used in this paper can be tailored to a city of any size. As such, a city is divided up into concentric rectangles of which sizes vary from one to three level 3 NAC cells, each NAC cell containing approximately $30 \times 30$ plots of $20 \times 50 \mathrm{~m}^{2}$. In this paper, a conversion algorithm is proposed to compute the postcode back and forth to NAC
\end{abstract}

Key words: Geocode, global postcode, natural area code, postal code, postcode, standard postcode.

\section{Introduction}

In the eighteenth century, the expansion of cities and the development of urban planning induced the need of having a way of locating properties. Initially the location only served as a distribution of property ownership in cities, but later on, new services emerged from the existence of addresses such as automated mail processing, vehicle navigation, urban planning and maintenance, emergency response, statistical analyses, marketing, etc. [1] As such, there was a need of creating coded delivery zones identified by letters and digits; these codes are referred to as postcodes and are an integral part of postal addresses.

The first postcode was established in Germany in early 1960s followed by USA and other countries [2]. At the beginning the creation of postcodes was solely intended for assisting in automated mail sorting and delivery. Afterwards, postcodes were recognized as a useful tool for cities and localities to allow people to 
easily find places. Postcodes were also adapted by different authorities to dispense services in a more efficient way. Postcodes, being geographical entities that are generally stable over time, also became a cornerstone to spatial strategic thinking and data collection and analysis [3]. Currently less than 150 out of 190 countries around the world have postcodes as part of their addressing systems [4] but there have not been standardized addressing schemes.

The International Standards Organization (ISO) is devising for a suite of international standards for addressing systems [5], [6], which can provide a common framework in order to benefit the economy, society and governance. In the effort to globalize the task of addressing, many codes such as what3words have been created. On the other hand, Posttudes, Global Postcode System and others were created with intention to be used as global postcode but none of them has been adopted as a standard postcode system.

The current paper presents a postcode design scheme, which can be used as a global postcode standard. The research aims to design a postcode which is easy for human to read, understand and give a sense of distance and orientation. The design also considers three criteria which a well-designed addressing system follows, namely human requirement, digital representation and physical representation.

\subsection{Problem Statement}

Lack of a global standard postcode scheme.

\subsection{Research Hypothesis}

To create a new postcode system based on the NAC, which can be efficiently used by humans.

\subsection{Research Objectives}

To propose a global standard postcode design scheme that can be adopted in any city whether structured or unstructured.

\section{Geocode Systems}

A geocode (Geographic entity object code) is a code used to identify a point or an area on the surface of the earth. The number of geocode systems exceeds 54 [7]. Most of them are proprietary systems with specific aims and utilities. The more natural and well-known geocode system is the Geographic coordinate system. In this system, any point on the earth is represented by its latitude and longitude. A very well-known application of the Geographic coordinate system is the Global Positioning System (GPS).

Although all geocodes are mainly used to locate places on the earth, most of them are not simple. Attempts have been made to simplify geocodes for human usage. For example, the Mapcode system is an innovative spatial positioning system built specially to make shorter codes for densely populated area in order to easily be recognized. Mapcode uses rectangular areas called "encoding zone", to define a location and uses letters and digits to represent territories within certain distance from that location. A 4-letter code can be used to cover a territory of $100 \mathrm{~km}^{2}$ and a 6-letter code can cover a 250,000 km² approximately. For

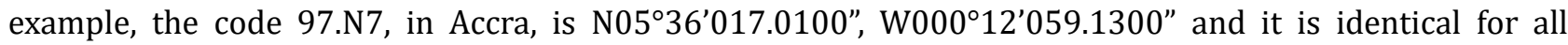
coordinates within 5 meters of this coordinate. Mapcode is very popular in car navigation systems and software are freely available to convert coordinates into Mapcode and vice versa [8], [9].

Another attempt to create simple geocode systems was the What3words system which aims at making easily memorable codes without using the common grouping of letters and digits. It is based on grids covering the earth of squares of $3 \times 3 \mathrm{~m}^{2}$ encoded using a format referred to as the $3 \mathrm{~m}$ format. The $3 \mathrm{~m}$ format is a combination of 3 words to uniquely identify each of the 57 trillion cells of the grid around the globe. E.g. the Eiffel Tower: graphics.dads.inched.

In this research, we are interested in a geocode that can be used as a global postcode scheme. The concept 
of a global geocode based postcode scheme is not new, for example, Posttude is a postcode scheme that can provide everyone on the earth with a postcode using Geotude (a mathematical algorithm that converts latitude and longitude into a single code) [10]. The Global Postcode (GPC) is another global postcode scheme that is derived from the Natural Area Coding (NAC) using a set of rules.

The NAC is a geocode system that can uniquely locate any property on the surface of the earth using an 8 to 10 alphanumeric code. A NAC consists of three alphanumerical strings separated by spaces representing respectively the longitude, the latitude and the altitude. Usually, the altitude is omitted and a NAC code represents an area on the earth. The NAC system divides the whole surface of the world into 30 divisions longitudinally and 30 divisions latitudinal. Each of these subdivisions forms the outer and biggest NAC cell (level one). NAC cells at level one are once again subdivided into a set of $30 \times 30$ cells using the same method to obtain smaller subdivisions (level two). It continues in the same way to get even much smaller subdivisions depending on the sought precision. Blocks at each level are named using numbers between 0 and 9 and 20 capital consonants of the English alphabet; vowels are excluded to avoid probable confusion between characters such as "0" (zero) and "O" (capital "o"), and " 1 " (one) and "I" (capital "i"). Names are assigned in an ascending order starting from numbers then alphabetic characters using the base 30 presented in Table 1, from the South to the North and from the West to the East [11].

First level NAC cells, which represent an area of approximately $1500 \times 750 \mathrm{~km}^{2}$ are represented by two characters separated by spaces (e.g., NAC: A 2). A second level NAC cell is represented by four characters (e.g., NAC: AC 12). In this code, characters $A$ and 1 are coordinates of the first level block which contains the second level cell represented by NAC: C 2 . To represent a block or a building NAC codes can be extended to 8 or 10 characters. (e.g., 7HGGKJ9L).

Although various other geocodes such as Mapcode and What3words tried to provide global addressing solutions; NAC simplifies the task of zoning, therefore can be used as postcodes. It can also provide property identifiers where street addresses are not in place.

Table 1. Base 30 Positional Numeral System

\begin{tabular}{llllllllllll}
\hline \hline Decimal & 0 & 1 & 2 & 3 & 4 & 5 & 6 & 7 & 8 & 9 \\
\hline Base 30 & 0 & 1 & 2 & 3 & 4 & 5 & 6 & 7 & 8 & 9 \\
\hline \hline Decimal & 10 & 11 & 12 & 13 & 14 & 15 & 16 & 17 & 18 & 19 \\
\hline Base 30 & $\mathrm{B}$ & $\mathrm{C}$ & $\mathrm{D}$ & $\mathrm{F}$ & $\mathrm{G}$ & $\mathrm{H}$ & $\mathrm{J}$ & $\mathrm{K}$ & $\mathrm{L}$ & $\mathrm{M}$ \\
\hline \hline Decimal & $\mathrm{20}$ & 21 & 22 & 23 & 24 & 25 & 26 & 27 & 28 & 29 \\
\hline Base 30 & $\mathrm{N}$ & $\mathrm{P}$ & $\mathrm{Q}$ & $\mathrm{R}$ & $\mathrm{S}$ & $\mathrm{T}$ & $\mathrm{V}$ & $\mathrm{W}$ & $\mathrm{Y}$ & $\mathrm{Z}$ \\
\hline \hline
\end{tabular}

\section{Addressing Needs and Postcode Systems}

A postcode is a key constituent of an address, also known in different countries as postcode, post code, ZIP code, PIN code, Eircode and other names [12], [13]. A postcode is made up of digit and/or letters added to a postal address to identify places and to increase the speed and accuracy of sorting and distributing mails. In some cases, there is no differentiator for addresses; as such, postcode becomes very essential to remove the ambiguity of addresses. For example, in Canada 12 Gibson Ave, Toronto" represents three different locations very distantly apart. The postcodes M4W2L4, M9V 2S5 and M9N1G1 would be required at this point. The Canadian postcode is an alphanumeric string in the form of ANA NAN; A representing alphabetic characters and $\mathrm{N}$ representing numeric characters. The Canadian postcode is made up of two parts: Forward Sortation Area (FSA) and Local Delivery Unit (LDU) (see Fig. 1). 


\section{$\widehat{\text { K1A OB1 }}$ \\ Forward
$\begin{gathered}\text { Local } \\ \text { Sortation } \\ \text { Area }\end{gathered}$
Delivery
Unit}

Fig. 1. Components of a Canadian postcode.

Forward Sortation Area (FSA) uniquely represents the province or territory. Each province or territory of 18 major territories or provinces is identified by the first digit and it represent postal district, For Example: A Newfoundland and Labrador, B Nova Scotia, C Prince Edward Island, E New Brunswick G Québec East H Montréal. The second digit of FSA denotes a rural Postcode when it is 0 and an urban Postcode area for other digits. The third digit is used to precise an area of the city or town. The Local Delivery Unit (LDU) representing the last three digits of the Postcode indicates a specific single postcode, which can correspond for example to a single block, an apartment or business building, a community mailbox. FSA and LDU contain over 835,000 unique and 1,100,000 multiple location Postcodes [14], [15].

In addition to making more efficient the sorting and delivery of mails, postcodes or ZIP in USA or Canada are used for a range of other purposes, for example, marketing, mapping software, online tools to help find information and services.

Various countries have postal address systems in place for decades to aid in sorting mails. With time, there has been a need of changing those systems to efficiently provide numerous other services and reflect the changes brought by the complexity growth of cities. For example, the American ZIP code (Zone improvement Plan Code) was started in 1963 to make quick and simple the process of delivering mails. The ZIP consisted of 5 digits which could identify a delivery area. (e.g. the Washington Square Park Zip Code is 10011). The first digit of the ZIP code indicates a region of the country. The next two digits indicate a sectional center facility (SCF) which essentially is the sorting operator. The last two digits indicate the post office used to deliver the mails. After 20 years, the US postal service (USPS) extended the ZIP to become $\mathrm{ZIP}+4$ (e.g. Blossom Baker Code is 10011-3363), the last four digit usually omitted are designated to point directly to an address such a house or a city block.

However, the ZIP code does not always reflect the area you are in. In some cases, the SCF of certain regions do not line up with the sequence of the ZIP code. For example, the SCF in ZIP code might reference the endpoint of an itinerary situated many miles away, even though we are just at the beginning of the route. Another limitation is that the U.S. postal service (USPS) developed the zip code system only to improve mail delivery, not a system to provide other services such as markets mapping [16]. Although the ZIP code is specific to the United States of America, some other countries like Singapore, India, UK postcode etc. have similar systems.

Newer postcodes are emerging with aim to address several problems built into the older systems. For example, the Ireland postcode (Eircode) released for public use in 2015 [17]. It provides a unique code for every address in Ireland. Although Eircodes is newer, it uses a database to be associated to Geo-coordinates. Also, Eircodes are random and codes in the same area are not sequential, thereby less meaningful. Also, Eircode is not easy to remember, communicate and it would not provide sense of distance or orientation to the user.

Around 40 countries who are members of the Universal Postal Union (UPU) do not have a postcode systems. Because implementing a proper postcode system is a long and expansive process. A universal postcode system based on a geocode could provide a low-cost solution [18]. Being the first application of NAC, GPC was an attempt to create a postcode scheme to be used globally, but one of the problems is that people cannot easily remember GPC because codes do not have local references.

Having a postcode in place can benefit citizens despite social economical classes [19]. Designing an effective postcode scheme is not fairly an easy effort. Some postcodes can be rather confusing or are less 
well-organized. A good design must remove the ambiguity of identifying an address, it must be unique, easily readable and give the sense of direction and distance [20].

Today the economic value and benefits of having an established postcode linked to a geocode cannot be underestimated. For example, private or government institutions which require usage of mapping software can benefit from the postcode geodata, for example, geographical grouping or labeling areas of high risks or to map demographic information and use Geo-demographical model to estimate the socio-economical profile [16].

In this paper, we propose a postcode scheme based on an internationally recognized geocode (NAC), which would work as a universal postcode and be an effective way to bring about the expansion of the global addressing system. Natural Area Code (NAC) is used herein because of its levels of acceptance, simplicity and integration.

\section{Postcode Design}

In this section, a postcode design scheme that can be used by any country that needs to establish a new postcode system or want to upgrade an existing postcode system is proposed.

\subsection{Postcode Format}

The format of the proposed postcode is AX NONON N XXXX, where ' $\mathrm{A}$ ' indicates an alphabetic character, ' $\mathrm{N}$ ' a numeric character, ' $\mathrm{X}$ ' an alphanumeric character and ' $\mathrm{O}$ ' indicates a direction. ' $\mathrm{O}$ ' can only take values ' $\mathrm{N}$ ' for North, 'E' for East, 'W' for West and ' $\mathrm{S}$ ' for South. The postcode is made up of components specified as follows:

AX: Indicates a reference point in a city from which distances are measured and orientations are given. Ideally the character ' $\mathrm{A}$ ' will be the initial letter, which represents a city or town's name, for example $\mathrm{K}$ for Kigali. Given that more than one city in a country could have the same name, and one city could have more than one reference points. The second character ' $\mathrm{X}$ ', which is an alphanumeric character, is used to distinguish names of cities within a country or different reference points within a city.

NONON: Indicates a postcode zone. The first two characters ' $\mathrm{NO}$ ' indicate a distance and direction measured from the reference point and the second part 'NO' specifies a distance and direction taken perpendicularly from the first distance. The fifth character ' $\mathrm{N}$ ' identifies the postcodes zone inside the orientation zone. $\mathrm{N}$ : An optional number added to ensure that provided directions are accurate. For example, NW and WN are in the same quadrant and indicates same directions but are in two different regions of the quadrants. Regions and the corresponding numbers are illustrated in Fig. 2.

XXXX: The last part of the postcode is also an optional component required only if there is no other addressing system in place; it can serve as house number, an apartment number or a block number. Only two characters 'XX' are sufficient if a level 4 NAC code can uniquely locate the property within an area of $25 \times 50 \mathrm{~m}^{2}$. In case a level 4 cell cannot locate the property precisely; a level 5 NAC code is used. Thus, two more characters are added. While these characters can serve as property numbers, they correspond to the last two or four characters of the level 4 or level 5 NAC coordinates respectively.

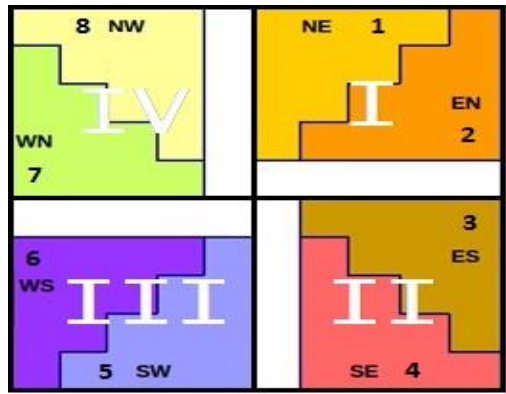

Fig. 2. Quadrants and regions. 


\subsection{Postcode Zones}

The technique proposed in this design consists of four main steps. (1) Choosing a reference point. (2) Defining distance zones from the reference point. (3) Dividing distance zones into orientation zones. (4) Dividing orientation zones into postcode zones.

Reference point: Initially a reference point is chosen, e.g. a city center monument, a landmark place or an emblematic object. A reference point is related to a section of the city or could be related to a whole city. More than one reference points can be used depending on the size of the city. A level 3 NAC code is then assigned to each reference points. E.g., Kigali is a relatively small city and a single reference point such as the main roundabout might be enough; its NAC is KH4 GN8.

Distance Zones: Distance zones are divisions done by concentric rectangles centered at the reference point, they are defined following the same logic as Amin's [20]. The separation distance between two consecutive rectangles depends on the length and the width of the smallest rectangle that fully covers the city or a section of the city. The separation distance can have three different dimensions: a single level 3 NAC cell, a block of $2 \times 2$ or $3 \times 3$ level 3 NAC cells. In all three cases, each of the level 3 NAC cells represents a postal zone, which is identified by digits from '0' to ' 9 '; therefore, the dimensions of blocks are limited to $3 \times 3$ cells. A bigger block like $3 \times 4$ or $4 \times 4$ will require more characters. Also, distance zones are named using digits from ' 0 ' to ' 9 ', therefore, a maximum of 10 orientation zones from the inner rectangle to the outer rectangle is allowed. Fig. 3 illustrates how distance zones are configured.

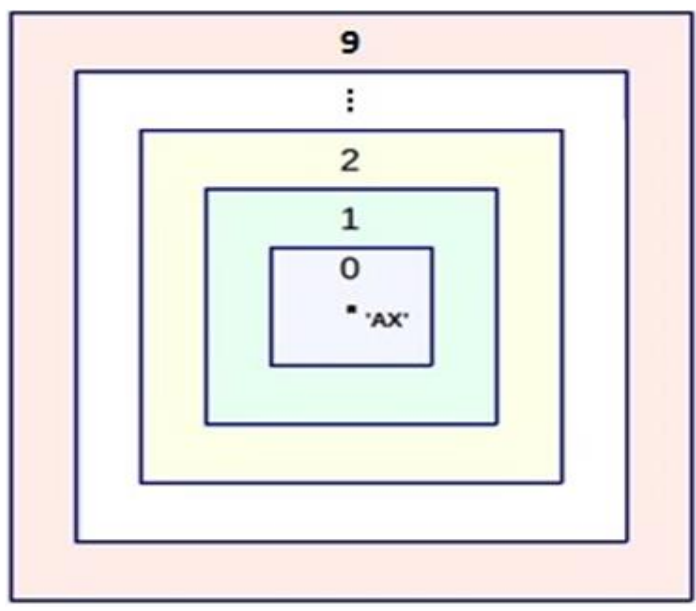

Fig. 3. Quadrants and regions.

The approximate maximum areas that can be covered depending on the size of the orientation zones are shown in Table 2.

Table 2. Base 30 Positional Numeral System

\begin{tabular}{cc}
\hline \hline Separation distance & Covered area (Width $\times$ Length) \\
\hline 1 NAC cell & $7.5 \times 15 \mathrm{~km} 2$ \\
\hline $2 \times 2$ NAC cells & $15 \times 30 \mathrm{~km} 2$ \\
\hline $3 \times 3$ NAC cells & $22.5 \times 45 \mathrm{~km} 2$ \\
\hline \hline
\end{tabular}

Orientation zones: Each of the distance zones is divided into orientation zones. Two levels of orientation zones are defined as follows:

Level one orientation zones are subdivisions of distance zones into Northern, Southern, Eastern and Western parts as illustrated in Fig. 4. 


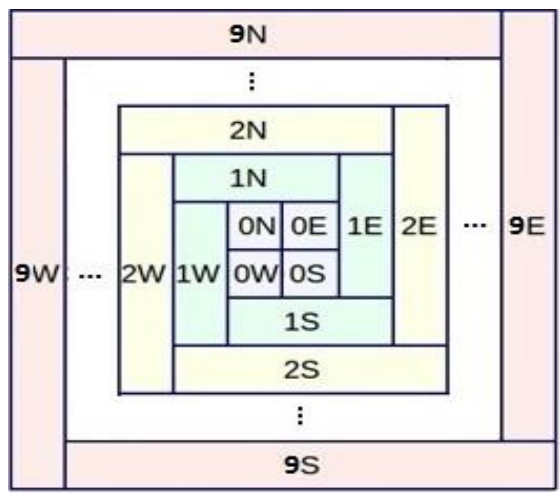

Fig. 4. Level one orientation zones.

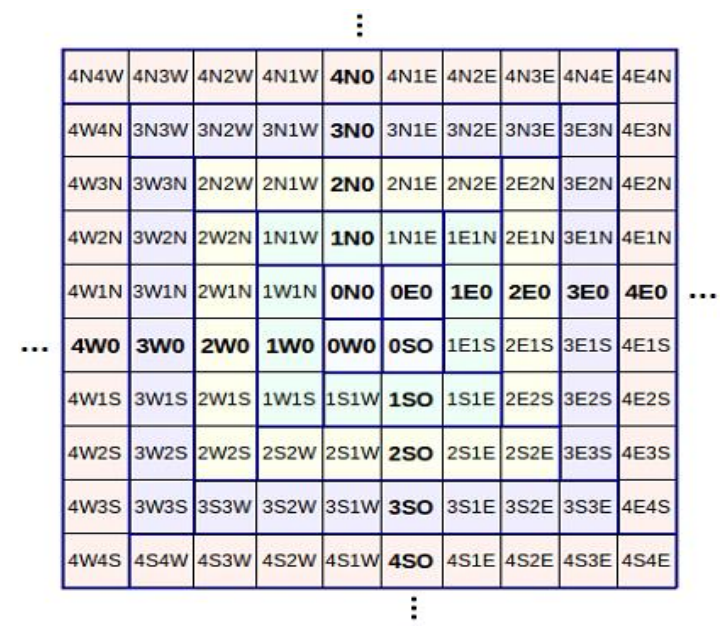

Fig. 5. Level two orientation zones.

Level two orientation zones are obtained by dividing each of the first level zones into blocks of $1 \times 1,2 \times 2$ or $3 \times 3$ level 3 NAC cells depending on the width of the first level zones. The obtained number of blocks equals to $2 n+1$, where $\mathrm{n}$ is the distance zone. Second level orientation zones are numbered from the middle zones of the first level zones called reference zones. Reference zones are named zero ' 0 ' and divide the city or town into 4 quadrants. In each first level zone, the numbering of second level zones increase gradually by one as you move away in any directions from the reference zone. An illustration of level two orientation zones is given in Fig. 5.

For example, in the first level orientation zone 3N the reference zone is 3N0; Separating West and East. From N0 to West or East 0 is replaced by 2 characters indicating the direction and distance, for example, $2 \mathrm{~N} 1 \mathrm{~W}$ is in the second Northern distance zone at level one in and in the first Western orientation zone at level two.

Postcode zones: The last step of the design is to locate postcode zones inside orientation zones, recalling that each postcode zone has the size of a level $3 \mathrm{NAC}$ cell. Its location depends on the size of the orientation zone. Three cases are possible and postcodes are defined in each case as follows:

Case 1: One 3rd level NAC cell orientation zone. In this case the size of the orientation zone is the same as the size of one postcode zone and it is identified by character ' 0 '; however, this character ' 0 ' might be removed to reduce the number of postcode characters.

Case 2: $2 \times 2$ rd level NAC cells orientation zone. In this case, one orientation zone consists of 4 postcode zones identified by numbers from ' 0 ' to ' 3 '.

Case 3: $3 \times 3$ level 3 NAC cells orientation zone. In this case, one orientation zone encloses 9 postcode 
zones. They are named after numbers from '0' to ' 8 '. To ensure uniformity in codes, each unit is assigned an ordered pair of numbers as shown in Table 3.

Table 3. Pairs and their Corresponding Number

\begin{tabular}{ccccccccc}
\hline \hline 0 & 1 & 2 & 3 & 4 & 5 & 6 & 7 & 8 \\
\hline$[0,0]$ & {$[0,1]$} & {$[1,0]$} & {$[1,1]$} & {$[0,2]$} & {$[2,0]$} & {$[1,2]$} & {$[2,1]$} & {$[2,2]$} \\
\hline \hline
\end{tabular}

The numbering of each pair prioritizes the North/South coordinate over West/East coordinate. Graphically these pairs of numbers represent intersections of coordinates. Fig. 6 shows how zone names are assigned inside an orientation zone.

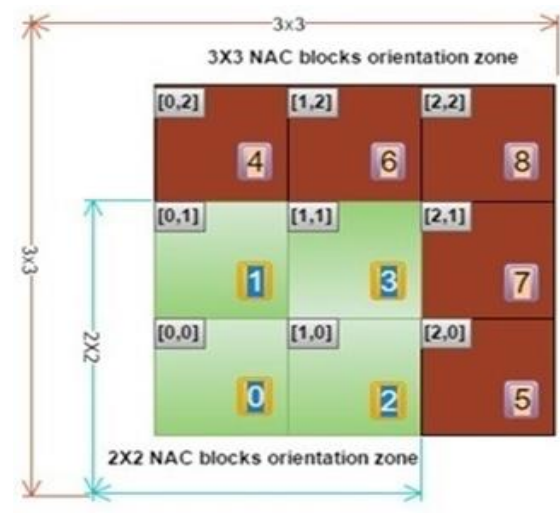

Fig. 6. Postcode zones inside an orientation zone.

The reference postcode zone ' 0 ' in the orientation block must be the closest zone to the reference zone. Therefore, postcode zones are placed in central symmetry with regard to the reference point as illustrated in Fig. 7.

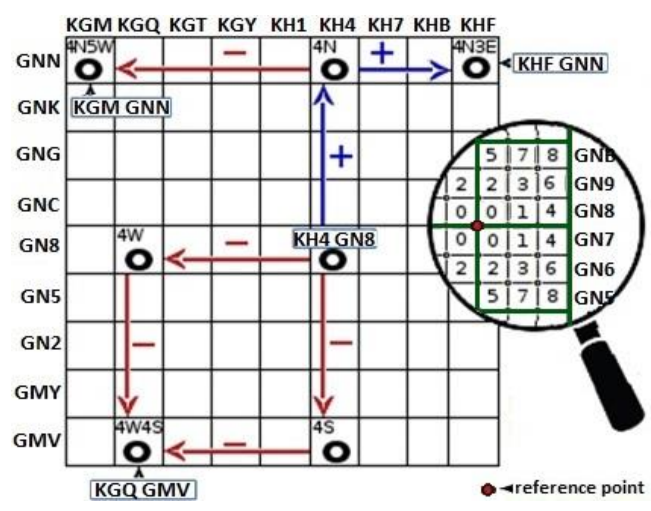

Fig. 7. Postcode zones configuration.

\section{Analysis of the Postcode}

Postcode being an integral part of an addressing system, the analysis of the postcode designed in this paper takes into consideration on three requirements, which a well-designed addressing system should satisfy; namely the human requirements, the digital representation and the physical representation [19].

\subsection{Human Requirements}

The designed postcode is: 
1) Easy to read: Composed of meaningful characters Initials of cities, orientation characters (N, E, S, O) and digits from 0 to 9 which indicate distance.

2) Short: The shorter the size of the postcode the better for human to remember. For small cities or towns, orientation zone blocks could correspond to a single postcode unit, making the postcode as short as 6 digits, for example, KN2N3W. Any city regardless of its size can be divided into small sections with different reference points and have short postcodes. Otherwise, depending on the size of the city, orientation blocks could be made up of 4 or 9 postcode units and the postcode size will increase by 1 digit, which identifies the postcode unity inside the orientation block.

3) Meaningful: It provides the ability to locate postcode units, that is, estimates distance and orientation from a reference zone.

Uniform: Besides the reference point, zone codes (for example 2N3W) are identical for any cities in the world that implement this postcode; making it easy for human to navigate whether in their home or foreign countries.

Orientation: 2N3W is in North-West from the reference point

Sequential: Codes vary per the distance and direction. Thus, the next postcode can be predicted based on distance and direction.

Distance: From the reference point the postcode unit $5 \mathrm{~N} 5 \mathrm{~W}$ is far-off compared to $2 \mathrm{~N} 3 \mathrm{~W}$ the distance between two postcode zones can be estimated using the postcode based distance calculation or using NAC based distance estimation (see digital representation section below).

Distance calculation: This approach uses human intuition and easy approximations to calculate distance, within the same section of the city, based on the numbers and orientations provided in the postcode.

Example: Distance between KG4W4S2 and KG3N5E3, where orientation block is $3 \times 3$, is calculated by adding the Southern distance $4 \mathrm{~S}$ to the Northern distance $3 \mathrm{~N}$, which gives 7 units of distance and by adding Western distance $4 \mathrm{~W}$ to Eastern distance 5E, which is 9 units of distance from West to East. Considering the length of one unit to be estimated to $4.5 \mathrm{~km}$ and width to be $2.25 \mathrm{~km}$ approximately, the indirect line distance $d 1$ is be calculated as

$$
d 1=7 \times 2.25 \mathrm{~km}+9 \times 4.5 \mathrm{~km}=56.25 \mathrm{~km}
$$

Using the Pythagorean distance formula, we can estimate the straight-line distance between the two postcode zones $d 2$ as

$$
d 2=\sqrt{(7 \times 2.25)^{2}+(9 \times 4.5)^{2}} \mathrm{~km}=43.45 \mathrm{~km}
$$

The difference between the two distances estimated using the direct and indirect lines may appear to be big, but roads and streets do not always follow straight paths; therefore, both distances are significant depending on the usage, the first one gives a more realistic estimation to a traveler and the direct line distances could be useful for map users who wish to know the approximate distance.

\subsection{Digital Representation}

Being based on NAC, this postcode naturally integrates with mapping systems like Google Maps. No need for a database to convert the code back and forth to NAC, rather an algorithm is used.

1) Conversion algorithm: The algorithm is a function $f$ of three variables, the reference point $r=N A C$ : $\mathrm{XXX}_{1} \mathrm{YYY}_{1}$, the size of the orientation zone $\mathrm{d}$, and the postcode $\mathrm{p}=$ NONON, $\mathrm{f}(\mathrm{r}, \mathrm{d}, \mathrm{p})$ computes back to the corresponding NAC. Considerations:

- The reference point is always assigned to the first quadrant; 
- As NACs increase in the North and East directions and decrease in South and West directions, each direction corresponds to a sign as shown in Table 4.

Table 4. Directions and Corresponding Signs

\begin{tabular}{ccc}
\hline \hline Longitude & Latitude & Sign \\
\hline North $(\mathrm{N})$ & East $(\mathrm{E})$ & + \\
\hline South $(\mathrm{S})$ & West $(\mathrm{W})$ & - \\
\hline \hline
\end{tabular}

Step 1: $\mathrm{NONON}$ is divided into 3 parts $\mathrm{N}_{1} \mathrm{O}_{1}, \mathrm{~N}_{2} \mathrm{O}_{2}$, and $\mathrm{N}_{3}$.

Step 2: Depending on the quadrant in which the post code is located, the reference point will be adjusted as follow:

Quadrant 1: the reference point remains unchanged;

Quadrant 2: the latitude part of the reference point decreases by one;

Quadrant 3: both the latitude and longitude parts of the reference point decrease by one;

Quadrant 4: the longitude part of the reference point decrease by one.

Step3: If $\mathrm{O}_{1}$ represents a longitudinal direction, $\mathrm{O}_{2}$ will represent a latitudinal direction; is added or subtracted to Latitude part of the postcode and is added or subtracted to the longitudinal part of the postcode or vice versa. The result yields a NAC of the orientation zone NAC: $\mathrm{XXX}_{2} \mathrm{YYY}_{2}$, where $\mathrm{XXX}_{2}$ is the longitude and $\mathrm{YYY}_{2}$ is the latitude,

Step4: $\mathrm{N}_{3}$ corresponds to a pair of coordinates $\left[\mathrm{C}_{1}, \mathrm{C}_{2}\right]$ provided in Table 3 . To find the actual NAC corresponding to the postcode depends on the quadrant.

Quadrant 1: the first coordinate $\mathrm{C}_{1}$ is added to $\mathrm{XXX}_{2}$ and the second coordinate $\mathrm{C}_{2}$ is added $\mathrm{YYY}_{2}$.

Quadrant 2: the first coordinate $\mathrm{C}_{1}$ is added to $\mathrm{XXX}_{2}$ and the second coordinate $\mathrm{C}_{2}$ is subtracted from $\mathrm{YYY}_{2}$.

Quadrant 3: the first coordinate $\mathrm{C}_{1}$ is subtracted to $\mathrm{XXX}_{2}$ and the second coordinate $\mathrm{C}_{2}$ is subtracted from $\mathrm{YYY}_{2}$.

Quadrant 4: the first coordinate $\mathrm{C}_{1}$ is subtracted to $\mathrm{XXX}_{2}$ and the second coordinate $\mathrm{C}_{2}$ is added from $\mathrm{YYY}_{2}$. The conversion algorithm is represented in Fig. 8.

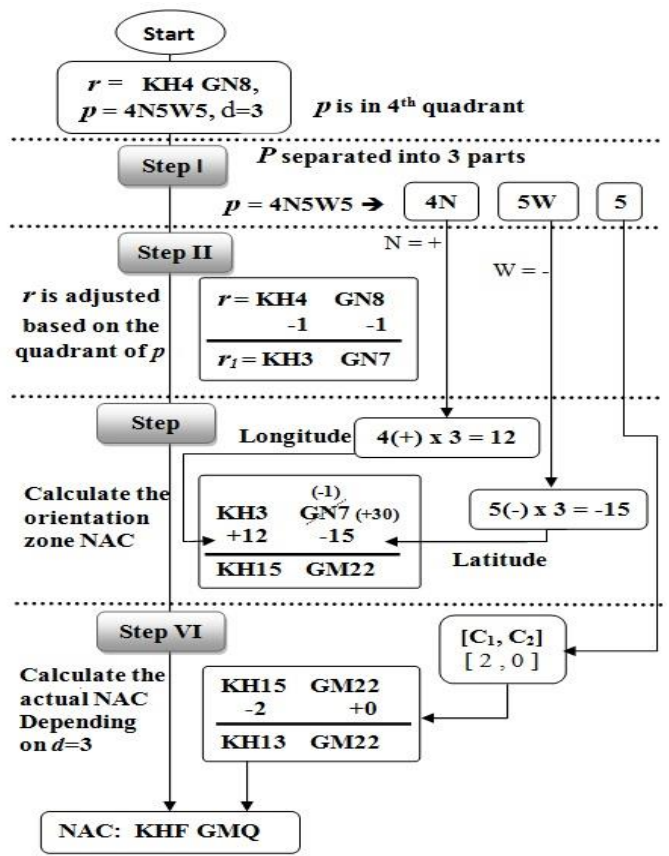

Fig. 8. Postcode to NAC conversion algorithm. 


\subsection{Physical Factors}

1) Easy to implement: Given that NACs can be found by zooming and panning maps using tools such as Google Maps API. Also, given that the algorithm proposed in this research can be used to convert NACs into postcode, the cost of rolling out a new postcode system will be minimal.

2) Uniquely identify properties: In area where there are no named streets and house numbers, the designed postcode can be used to designate a property as small as a house. Thereby, allowing each property to possess a unique address.

\subsection{Weaknesses and Threats}

1) Long if all the digits are used: The postcode size in this research varies depending on the usability and needed precision. In the case, where all features of the code are used, the size of the postcode reaches 12 digits.

2) Administrative structures: The postcode structure does not follow administrative divisions and hierarchies. The districts, municipality names or initials are not necessary reflected in the postcode.

3) Postcode delimitation: Natural delimitations like rivers, forest etc. or roads, which are references for humans, are not considered.

4) Similar postcode: There exists other postcodes universally well-advertised and accepted.

\section{Conclusion}

In this paper, several existing postcodes are reviewed and their strengths and weaknesses are discussed. Common issues in some of existing postcodes have been highlighted, among others the implementation cost, the need of maintenance/adaptation and integration with new technologies.

The postcode proposed in this research is based on the Natural Area Coding, in this way, the cost of implementation is practically low; also, the postcode is naturally integrated with mapping tools and can be uniquely be assigned to properties in any type of cities from well planned to slums. Using an algorithm, the postcode can be converted back and forth to NAC giving it a better performance.

The postcode is easy for human usage, it uses local references, gives a sense of distance and orientation and it is sequential. The robustness of the designed postcode will be demonstrated through future works, which will include the conversion software design and the implementation of the postcode.

\section{References}

[1] Joint Committee on Communications, Energy and Natural Resources. (2010). Annual Report 2007-2009. House of the Oireachtas, Dublin.

[2] Lombaard, M. South African Postcode Geography. The Pennsylvania State University CiteseerX Archive.

[3] Norman, P. D. (2016). Georeferencing Socio-Demographic Information: Postcode, Addresses, Land Parcels and Geographic Information Systems. University of Leeds, Tech.

[4] UPU. (2016). Looking up a postcode. Retrieved August 2016, from www.upu.int/en/resources/postcodes/looking-up-a-postcode.htm

[5] Coetzee, S., Cooper, A. K., Lind, M., Wells, M. M., Yurman, S. W., Griffiths, N., \& Nicholson, M. J. (2008). Towards an international address standard. Proceedings of the 10th International Conference for Spatial Data Infrastructure.

[6] Cooper, A. K. \& Coetzee, S. (2008). The South African address standard and initiatives towards an international address standard. Proceedings of the Academic Track of the 2008 Free and Open Source Software for Geospatial (FOSS4G) Conference.

[7] Wikipedia. (2016). Category: Geocodes. Retrieved August 2016, from 
https://en.wikipedia.org/wiki/Category:Geocodes

[8] Potgieter, P. (2014). Mapcodes: A new standard for representing locations. EE Publishers.

[9] Geelen, P. (2015). Mapcode: The Netherlands a public location reference standard. Distribution Courtesy of the Mapcode Foundation.

[10] Abas, R. (2010). Ever decreasing squares. Postal Technology International.

[11] N. T. G. Inc. (2016). The natural area coding system. Retrieved August, 2016, from http://www.nacgeo.com/nacsite/documents/nac.asp

[12] UPU S42. (2006). S42: International postal address components and templates. Universal Postal Union (UPU).

[13] Walker, R. (2008). A general approach to addressing. ISO Workshop on Address Standards: Considering the Issues Related to an International Address Standard, 23-27. University of Pretoria, Copenhagen, Denmark.

[14] Canadian Post. (2016). Addressing guidelines, Canada postal guide. Retrieved January 2016, from https://www.canadapost.ca/tools/pg/manual/PGaddress-e.asp

[15] FADAIE, K. (2000). A framework and reference model. International Archives of Photogrammetry and Remote Sensing, XXXIII(PartB2).

[16] Office of Inspector General. (2013). The untold story of the zip code. U.S. Postal Service, Tech.

[17] Eircode. (2015). Code of Practice.

[18] Post \& Parcel. Addressing the world: How geocodes could help billions start using the mail. Retrieved November

2011 ,

from

http://postandparcel.info/43564/in-depth/addressing-the-world-how-geocodes-could-help-billions-s tart-using-the-mail/

[19] Coetzee, S., Cooper, A. K., \& Ditsela, J. (2011). Towards good principles for design of a national addressing scheme. Proceedings of the 25th International Cartographic Conference (ICC 2011).

[20] Amin, S. M., \& Wilson, D. (2014). Designing a postcode system for Arbil city Iraq. International Journal of Computer and Communication Engineering, 3(5).

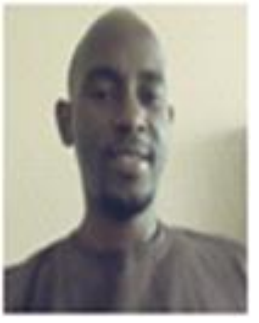

Valentin Rwerekane is working as a lecturer in the School of ICT, Department of Computer Science at the University of Rwanda, since 2015. He holds a master's degree in computer science from the University of Surrey, United Kingdom from 2009 to 2010. He has previously worked as freelance software developer in UK as well as in Rwanda.

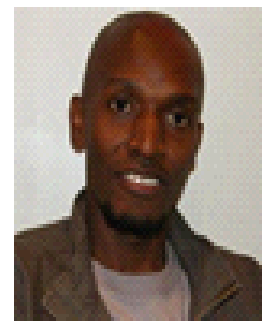

Maurice Ndashimye is a Ph.D candidate at iThemba Labs in South Africa, awarded a postgraduate diploma in mathematical science in 2011 at the African Institute for Mathematical Sciences in South Africa and an MSc. Eng. in mechanical engineering in 2015 at the University of Stellenbosch in South Africa. His research focuses on mathematical modeling and optimization of natural systems. 